\title{
POSITIVE SOLUTIONS OF AN ASYMPTOTICALLY PLANAR SYSTEM OF ELLIPTIC BOUNDARY VALUE PROBLEMS
}

\author{
F.J.S.A. CORRÊA \\ Departamento de Matemática e Estatística \\ Universidade Federal da Paraiba \\ 58109-970-Campina Grande-PB, BRASIL \\ e-mail julio@dme.ufpb.br
}

(Received August 10, 1995 and in revised form December 12, 1997)

\begin{abstract}
We will prove an existence result of positive solutions for an asymptotically planar system of two elliptic equations. It will be used as main tools for a Maximum Principle and a result on Bifurcation Theory
\end{abstract}

KEY WORDS AND PHRASES: Positive solution, asymptotically planar, maximum principle, bifurcation point.

AMS SUBJECT CLASSIFICATION CODES: $35 \mathrm{~J} 25$, 47H15.

\section{INTRODUCTION}

In this paper we will prove the existence of positive solutions for the elliptic system

$$
-\Delta U=A(x) U+F(x, U) \text { in } \Omega, U=0 \text { on } \partial \Omega
$$

where $\Omega \subset \mathbb{R}^{N}$ is a bounded smooth domain, $A(x)=\left(\begin{array}{ll}a(x) & b(x) \\ c(x) & d(x)\end{array}\right)$ whose entries are continuous in $\bar{\Omega}$, $U=\left(\begin{array}{l}u \\ v\end{array}\right),-\Delta U=\left(\begin{array}{cc}-\Delta & 0 \\ 0 & -\Delta\end{array}\right)\left(\begin{array}{l}u \\ v\end{array}\right)=\left(\begin{array}{l}-\Delta u \\ -\Delta v\end{array}\right) \quad$ and $\quad F(x, U)=\left(\begin{array}{l}f(x, u, v) \\ g(x, u, v)\end{array}\right) \quad$ with $f, g: \bar{\Omega} \times\left(\mathbb{R}^{+}\right)^{2} \rightarrow \mathbb{R}$ locally lipschitzian continuous satisfying.

$$
f(x, 0,0)>0 \text { or } g(x, 0,0)>0 \text { for all } x \in \bar{\Omega}
$$

and there is a positive constant $C$ so that

$$
0 \leq f(x, u, v), g(x, u, v) \leq C \text { for all }(x, u, v) \in \bar{\Omega} \times\left(I R^{+}\right)^{2} .
$$

Condition (3) says that the function

$$
\widehat{F}(x, U)=\left(\begin{array}{l}
a(x) u+b(x) v+f(x, u, v) \\
c(x) u+d(x) v+g(x, u, v)
\end{array}\right)
$$

is of asymptotically planar type. Since we are concerned with the existence of positive solutions we will suppose through this work that system (1) is cooperative, i.e., $b(x)$ and $c(x)$ are both nonnegative for all $x \in \bar{\Omega}$. This cooperativeness is imposed in order we may use a Maximum Principle (MP in short) In particular we will deal with the one due to the author of this paper in collaboration with $M A S$ Souto [1]. Using this $(M P)$ and a result on Bifurcation Theory we prove the following:

THEOREM 1. If $a(x)<\lambda_{1}, d(x)<\lambda_{1}$ and if either 


$$
|a|_{\infty} \leq|d|_{\infty} \text { and } 1<\frac{2 \lambda_{1}}{2|d|_{\infty}+|b|_{\infty}+|c|_{\infty}}
$$

or

$$
|d|_{\infty} \leq|a|_{\infty} \text { and } 1<\frac{2 \lambda_{1}}{2|a|_{\infty}+|b|_{\infty}+|c|_{\infty}}
$$

then problem (1) possesses a positive (classical) solution

Here $|\cdot|_{\infty}$ denotes the usual sup norm, that is, $|u|_{\infty}=\sup _{x \in \bar{\Omega}}|u(x)|$ and $\lambda_{1}$ is the first eigenvalue of $-\Delta$ in $\Omega$ under Dirichlet boundary condition.

To tackle this theorem we proceed as follows: Since $f$ and $g$ are both defined only for $u, v \geq 0$ we ought to consider the extensions of $f$ and $g$, respectiveley

$$
f_{1}(x, u, v)=f(x,|u|,|v|) \text { and } g_{1}(x, u, v)=g(x,|u|,|v|)
$$

now defined for all $(x, u, v) \in \bar{\Omega} \times \mathbb{R}^{2}$ We now carry on by setting

$$
L=\left(\begin{array}{cc}
-\Delta-a(x) & 0 \\
0 & -\Delta-d(x)
\end{array}\right), \bar{F}(x, U)=\left(\begin{array}{l}
f_{1}(x, u, v) \\
g_{1}(x, u, v)
\end{array}\right), B(x)=\left(\begin{array}{cc}
0 & b(x) \\
c(x) & 0
\end{array}\right) .
$$

Fixing these notations we are going to pay attention to the following nonlinear eigenvalue problem

$$
L U=\lambda[B(x) U+\bar{F}(x, U)] \text { in } \Omega, U=0 \text { on } \partial \Omega
$$

where $\lambda>0$ is a real parameter and it will be proved the existence of a continuum $\Sigma \subset \mathbb{R}^{+} \times[C(\Omega)]^{2}$ of solutions $(\lambda, U)$ of $(4)$ that begins at $(0,0)$ and extends beyond the line $\{1\} \times[C(\Omega)]^{2}$ arising a solution of (1) which in view of the (MP), should be positive.

As we will show after proving Theorem 1 the motivation in studying problem (1) came of the scalar one

$$
-\Delta u=f(x, u) \text { in } \Omega, u=0 \text { on } \partial \Omega,
$$

where $f$ has a sublinear behavior.

\section{PRELIMINARY RESULTS}

In order to establish the (MP) we begin by fixing some notations. Let $\mathbf{X}$ be a Banach space ordered by the positive cone $\mathbf{K} \subset \mathbf{X}$ and $\widehat{L}: \mathbf{X} \rightarrow \mathbf{X}$ a linear operator. By a $(M P)$ to problem

$$
U=\widehat{L} U+F, U \in \mathbf{X}
$$

we mean the statement $F \geq 0$ (i.e. $F \in \mathbf{K}$ ) imply $U \geq 0$ whenever that $U$ is a solution of (6).

PROPOSITION 2 (Maximum Principle). Let $\widehat{L}: \mathbf{X} \rightarrow \mathbf{X}$ be a positive linear compact operator (positive means $\widehat{L}(\mathbf{K}) \subset K)$. Then (6) satisfies the $(M P)$ if the condition below holds true

$$
\{U \in \mathbf{X}, t \in[0,1], U=t \widehat{L} U\} \Rightarrow U=0 .
$$

Now we shall focus our attention on the problem

$$
L U=B(x) U+\bar{F}(x, U) \text { in } \Omega, U=0 \text { on } \partial \Omega,
$$

to prove the following:

THEOREM 3. If $a(x)<\lambda_{1}, d(x)<\lambda_{1}$ and if either

$$
|a|_{\infty} \leq|d|_{\infty} \text { and } 1<\frac{2 \lambda_{1}}{2|d|_{\infty}+|b|_{\infty}+|c|_{\infty}}
$$

or 


$$
|d|_{\infty} \leq|a|_{\infty} \text { and } 1<\frac{2 \lambda_{1}}{2|a|_{\infty}+|b|_{\infty}+|c|_{\infty}}
$$

then every solution of $(8)$ is positive and so is a solution of (1).

PROOF. We first observe that the extension $\bar{F}(x, U)$ is also nonnegative. Second we notice that the operator $L=\left(\begin{array}{cc}-\Delta-a(x) & 0 \\ 0 & -\Delta-d(x)\end{array}\right)$ has an inverse $L=\left(\begin{array}{cc}(-\Delta-a(x))^{-1} & 0 \\ 0 & (-\Delta-d(x))^{-1}\end{array}\right):[C(\bar{\Omega})]^{2} \rightarrow[C(\bar{\Omega})]^{2}$ which is compact and positive in view of $a(x), d(x)<\lambda_{1}$ in $\bar{\Omega}$ So we will analyze uniqueness for the problem

$$
U=t L^{-1} B(x) U, U \in[C(\Omega)]^{2}, t \in[0,1]
$$

that is equivalent to

$$
\left\{\begin{array}{ccc}
-\Delta u-a(x) u=t b(x) v & \text { in } & \Omega \\
-\Delta v-d(x) v=t c(x) u & \text { in } & \Omega \\
u=v=0 & \text { on } & \partial \Omega
\end{array}\right.
$$

By multiplying both sides of the first equation in (9) by $u$ and both sides of the second one by $v$ and integrating by parts we obtain

and

$$
\int|\nabla u|^{2}=\int a(x) u^{2}+t \int b(x) u v
$$

$$
\int|\nabla v|^{2}=\int c(x) u v+\int d(x) v^{2}
$$

Since $a, b, c$ and $d$ belong to $C(\bar{\Omega})$ one gets, thanks to both Holder's and Pioncare's inequalities,

and

$$
\int|\nabla u|^{2} \leq \frac{|a|_{\infty}}{\lambda_{1}} \int|\nabla u|^{2}+\frac{|b|_{\infty}}{2 \lambda_{1}}\left(\int\left[|\nabla u|^{2}+|\nabla u|^{2}\right]\right)
$$

$$
\int|\nabla v|^{2} \leq \frac{|c|_{\infty}}{2 \lambda_{1}}\left(\int\left[|\nabla u|^{2}+|\nabla v|^{2}\right]\right)+\frac{|d|_{\infty}}{\lambda_{1}} \int|\nabla v|^{2}
$$

Summing up these two inequalities and assuming that $|a|_{\infty} \leq|d|_{\infty}$ one has

$$
\int|\nabla u|^{2}+\int|\nabla v|^{2} \leq \frac{1}{\lambda_{1}}\left[\frac{2|d|_{\infty}+|b|_{\infty}+|c|_{\infty}}{2}\right]\left[\int\left(|\nabla u|^{2}+|\nabla v|^{2}\right)\right] .
$$

Since $1<\frac{2 \lambda_{1}}{2|d|_{\infty}+|b|_{\infty}+|c|_{\infty}}$ we conclude that $U=0$. We arrive at the same conclusion by assuming assumption (ii). Thus system (8) enjoys the (MP) and in view of $\bar{F}(x, U) \geq 0$ we have $U \geq 0$ and so it is a solution of (1).

We now enunciate a proposition, due to Rabinowitz [5], which is another tool in proving Theorem 1.

PROPOSITION 4. Let $X$ be a Banach space and suppose that $T: \mathbb{R}^{+} \times \mathbf{X} \rightarrow \mathbf{X}$ is a continuous map. Then the nonlinear eigenvalue problem $u=T(\lambda, u)$ possesses an unbounded continuum of solutions meeting $(0,0) \in \mathbb{R} \times \mathbf{X}$, if in addition, we suppose $T(0, u)=0$ for all $u \in \mathbf{X}$.

\section{MAIN RESULTS AND REMARKS}

We start this section proving Theorem 1 .

PROOF OF THEOREM 1. Set $\mathbf{X}=[C(\Omega)]^{2}$ endowed with the usual norm $|U|_{\infty}=|u|_{\infty}+|v|_{\infty}$ Hence $\mathbf{X}$ is a Banach space and, as we said before, $L^{-1}: \mathbf{X} \rightarrow \mathbf{X}$ is linear, compact and positive. So problem (4) is equivalent to the following functional equation in $\mathbb{R}^{+} \times \mathbf{X}$ : 


$$
U=\lambda\left[L^{-1} A(x) U+L^{-1} \tilde{F}(x, U)\right], \lambda \geq 0, U \in \mathbf{X},
$$

where $\tilde{F}(x, U)$ is the Nemytskii operator associated with the function $\bar{F}$, i.e., for each $U \in \mathbf{X}$ one has

$$
\tilde{F}(\cdot, U(\cdot))(x)=\bar{F}(x, U(x)) .
$$

Since $L^{-1} A$ and $L^{-1} \widetilde{F}$ are compact operators we are able to conclude the existence of an unbounded continuum $\Sigma$ of solutions of (10) beginning at $(0,0) \in \mathbb{R}^{+} \times \mathbf{X}$. If $(\lambda, 0) \in \Sigma$ then $\lambda=0$ because $f(x, 0,0)>0$ or $g(x, 0,0)>0$ Plainly $(0, U) \in \Sigma$ implies $U=0$. Thus $\Sigma$ meets $\{0\} \times[C(\Omega)]^{2}$ and $\mathbb{R}^{+} \times\{0\}$ only at $(0,0)$. Note that bootstraping these solutions, that at first sight belong only to $[C(\bar{\Omega})]^{2}$, we obtain classical solutions.

It is worthy to say that hitherto we cannot affirm that $\Sigma$ contains positive solutions. In spite of this we can say that a piece (or perhaps pieces) of $\Sigma$ contains only positive solutions. Indeed, if $\lambda \leq 1$ we may prove, reasoning as in the proof of Theorem 3, that every solution $U$ of problem (10) is positive. It rests to show that in fact $\Sigma$ reaches $\lambda=1$

Since $\Sigma$ is unbounded it may be unbounded with respect to $\lambda$, or with respect to $U$ or with respect to both $\lambda$ and $U$. If $\Sigma$ is unbounded in $\lambda$ then it crosses the line $\{1\} \times \mathbf{X}$ and so we find a solution $U$ of the problem (4) and in view of assumptions (i) and (ii) of Theorem 3 is positive and so is a solution of (1). We now suppose that if $(\lambda, U) \in \Sigma$ then $\lambda \leq 1$. Hence there is a sequence $\left(\lambda_{n}, U_{n}\right) \in \Sigma$ with $\lambda_{n} \leq 1$ and $\left|U_{n}\right|_{\infty} \rightarrow \infty$ Thus

$$
L U_{n}=\lambda\left[B(x) U_{n}+\bar{F}\left(x, U_{n}\right)\right] \text { in } \Omega, U_{n}=0 \text { on } \partial \Omega .
$$

Setting $W_{n}=\frac{U_{n}}{\left|U_{n}\right|_{\infty}}$ we obtain

$$
L W_{n}=\lambda_{n}\left[B(x) W_{n}+\frac{\bar{F}\left(x, U_{n}\right)}{\left|U_{n}\right|_{\infty}}\right] \text { in } \Omega, W_{n}=0 \text { on } \partial \Omega .
$$

Passing to a subsequence if necessary we obtain $\lambda_{n} \rightarrow \lambda_{0} \in[0,1], W_{n} \rightarrow W$ in $[C(\bar{\Omega})]^{2}$ and $L W_{n} \rightarrow \lambda_{0} B(x) W \quad$ As $L: D(L) \rightarrow[C(\bar{\Omega})]^{2}$, where $D(L)=\left\{U \in[C(\Omega)]^{2} ; L U \in[C(\Omega)]^{2}\right.$ and $U=0$ on $\partial \Omega\}$, is closed one has that $W \in D(L)$ and

$$
L W=\lambda_{0} A(x) W \text { in } \Omega, W=0 \text { on } \partial \Omega .
$$

Because $W_{n} \rightarrow W$ in $[C(\Omega)]^{2}$ and $\left|W_{n}\right|_{\infty}=1$ then $|W|_{\infty}=1$, i.e., $W$ is a nontrivial solution of the above problem. But, in view of $(M P)$ and $\lambda_{0} \leq 1, W \equiv 0$ which is absurd. Thus $\Sigma$ crosses $\{1\} \times \mathbf{X}$ and, by Theorem 3, such solution is positive and the proof of Theorem 1 is over.

REMARK 1. The proof of Theorem 1 rests heavily on the existence of a (MP) like the one contained in [1] We must observe that this $(M P)$ is valid for a more general elliptic operator. Indeed, if we consider uniformly elliptic operators in the divergence form

$$
L_{k}=-D_{\jmath}\left(a_{i j}^{k} D_{\imath} u\right)+D_{\jmath}\left(a_{j}^{k} u\right), k=1,2
$$

(the symbols of summation are implicit in the expressions) where coefficients are regular enough, $a_{\imath j}^{k}=a_{\imath j}^{k}$ and setting $\lambda_{1}\left(L_{k}\right)$ as being the first eigenvalue of $\left(L_{k}, H_{0}^{1}(\Omega)\right)$, the system

$$
\mathcal{L} U=A(x) U+F(x) \text { in } \Omega, U=0 \text { on } \partial \Omega
$$

where $\mathcal{L}=\left(\begin{array}{cc}L_{1} & 0 \\ 0 & L_{2}\end{array}\right)$, enjoys the $(M P)$ if

$$
(B(x) \xi, \xi)<\lambda_{1}\left(L_{k}\right)\left(\xi_{1}^{2}+\xi_{2}^{2}\right)
$$


for all $\xi=\left(\xi_{1}, \xi_{2}\right)$ and $x \in \bar{\Omega}$ Here $(.,$.$) is the usual inner product in \mathbb{R}^{2}$ and $B(x)=\left(\begin{array}{cc}0 & b(x) \\ c(x) & 0\end{array}\right)$ Note that the above condition provides the uniqueness required by the (MP) in [1]. So Theorems 1 and 3 remain valid, with slight modifications, if system (1) is considered with $-\Delta$ substituted by the nonselfadjoint operators $L_{1}$ and $L_{2}$

REMARK 2. At the outset of our motivations in studying problem (1) we had considered the following

$$
L u:=-\sum_{\imath, \jmath=1}^{N} a_{\imath \jmath}(x) D_{\imath \jmath} u+\sum_{\imath, \jmath=1}^{N} a_{\imath}(x) D_{\imath} u=f(x, u) \text { in } \Omega, u=0 \text { on } \partial \Omega
$$

where $L$ is a second order uniformly elliptic operator in $\Omega$ with real smooth coefficients satisfying $a_{\imath \jmath}=a_{j i}$ in $\bar{\Omega}$, for all $1 \leq i, j \leq N$, and $f: \bar{\Omega} \times \mathbb{R}^{+} \rightarrow \mathbb{R}^{+}$is a sublinear nonlinearity. It is to say, setting

$$
a_{0}(x)=\lim _{t \rightarrow 0^{+}} \frac{f(x, t)}{t}, a_{\infty}(x)=\limsup \sup _{t \rightarrow \infty} \frac{f(x, t)}{t}
$$

one must have

$$
\lambda_{1}\left(a_{0}\right)<1<\lambda_{1}\left(a_{\infty}\right)
$$

where $\lambda_{1}\left(a_{2}\right), i=0, \infty$, is the first eigenvalue of the linear eigenvalue problem

$$
L u=\lambda a_{\imath}(x) u \text { in } \Omega, u=0 \text { on } \partial \Omega .
$$

Condition (13) says that we are working with a sublinear problem, i.e, in case, for instance, $a_{0}$ and $a_{\infty}$ are constants the nonlinearity $f$ begins, above the straight line $\lambda_{1} t$ and at the end it remains below the same line.

In Brezis-Oswald [2] the authors consider $L=-\Delta$ and use Variational Methods by exploring the selfadjointness of $-\Delta$ and $f$ is not necessarily a smooth function. In fact $a_{0}(x)$ and $a_{\infty}(x)$ may take values $+\infty$ and $-\infty$, respectively, so we address the reader to Section 3 of [2] for the precise meaning of (13).

In de Figueiredo [3] problem (11) is studied under condition (13) where $L$ is a selfadjoint operator more general than $-\Delta$ but $f$ is a $C^{\alpha}$-function, $0<\alpha<1$, and $f(x, t)+K t$ is nondecreasing in $t$ for some $K \geq 0$. In this case the sub and supersolution method is used.

If $L$ is not necessarily selfadjoint problem (11) was studied by Costa-Gonçalves [4] under condition (13), still using the sub and supersolution technique. In the works quoted above the authors always show existence of a positive solution as well as give sufficient condition for uniqueness.

This scalar problem arises a very natural question: How can we formulate a sublinear problem like before when we take a system into account?

We think that the best motivation towards a more general situation is to consider the biharmonic problem because it brings up for attention a very simple system and from it we would deal a more general sublinear problem. More precisely we first analyze the simplest biharmonic problem

$$
\Delta^{2} u=m u+g(u) \text { in } \Omega, u=\Delta u=0 \text { on } \partial \Omega,
$$

that is, the biharmonic equation under the so called Navier boundary conditions. Here $m$ is a positive constant. Setting $v=-\Delta u, A=\left(\begin{array}{cc}0 & 1 \\ m & 0\end{array}\right), G(U)=\left(\begin{array}{c}0 \\ g(u)\end{array}\right)$ we get the system

$$
-\Delta U=A U+G(U) \text { in } \Omega, U=0 \text { on } \partial \Omega \text {. }
$$


Taking $g$ a bounded function then $f(u)=m u+g(u)$ would be sublinear if it begins at $t=0$ zero above $\lambda_{1}^{2} t$ and remains below $\lambda_{1}^{2} t$ for $t$ large enough. Note that this is the counterpart of condition (13) when we are dealing with $\Delta^{2}$ Observe that $\lambda_{1}^{2}$ is the first eigenvalue of $\Delta^{2}$ in $\Omega$ under Navier boundary conditions and the situation described above occurs, for instance, if $g(0)>0$ and $m<\lambda_{1}^{2}$.

REMARK 3. Now we are going to analyze the condition given in Theorem 1 for the system (15) In this case one has that $\frac{1+m}{2}<\lambda_{1}$ is a sufficient condition in order system (15) enjoys the (MP).

Next we will show that this condition leads to a sublinear problem related to (14). Let us suppose that $\frac{1+m}{2}<\lambda_{1}$

a) If $m \neq 1$ one has $(m-1)^{2}>0$ which implies $\frac{(1+m)^{2}}{4}>m$ and since $\lambda_{1}>\frac{1+m}{2}$ we get $\lambda_{1}^{2}>\frac{(1+m)^{2}}{4}>m$ and so we have a sublinear problem.

b) If $m=1$ then $\lambda_{1}>\frac{1+m}{2}=1$ and hence $\lambda_{1}^{2}>\lambda_{1}>1=m$. In this case we still have a sublinear problem.

Therefore we believe that conditions (i) and (ii) are two kinds of sublinearity conditions when we deal with a system of two equations.

ACKNOWLEDGMENT. This work was partially supported by CNPq/Brasil.

\section{REFERENCES}

[1] CORREA, F.J.S A and SOUTO, M.A.S., On maximum principle for cooperative elliptic systems via fixed point index. Nonlinear Analysis, Theory Methods and Applications, 26, 5 (1996), 997. 1006.

[2] BREZIS, H and OSWALD, L., Remarks on sublinear elliptic equations, Nonlinear Analysis, Theory Methods and Applications, 10, 1 (1986), 55-64.

[3] de FIGUEIREDO, D.G., Positive solutions of semilinear elliptic problems, Proc. of the 1st Latin American School of Differentıal Equations, São Paulo, Springer-Verlag (1981), 34-87.

[4] COSTA, D.G. and GONÇALVES, J.V.A., On the existence of positive solutions for a class of nonselfadjoint elliptic boundary value problems, Applicable Analysis, 31 (1989), 309-320.

[5] RABINOWITZ, P H., Some global results for nonlinear eigenvalue problems, J. Funct. Anal. 7 (1971), 487-513 


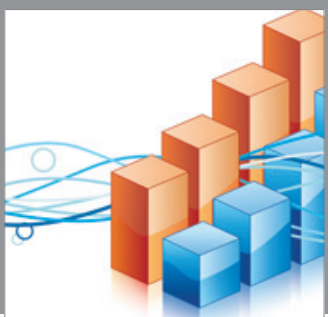

Advances in

Operations Research

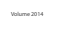

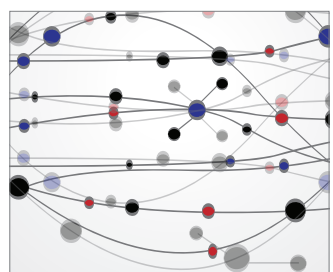

\section{The Scientific} World Journal
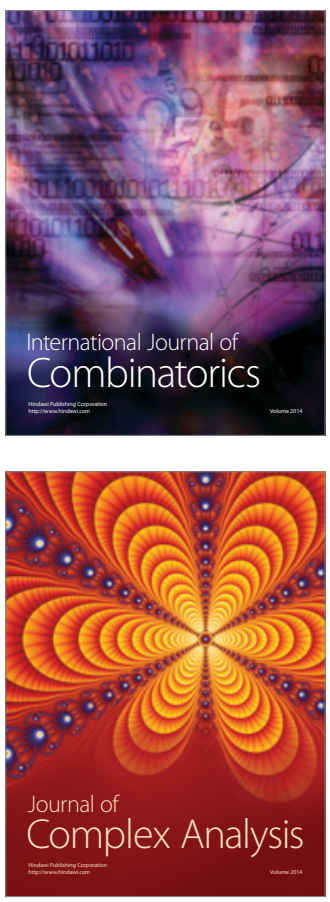

International Journal of

Mathematics and

Mathematical

Sciences
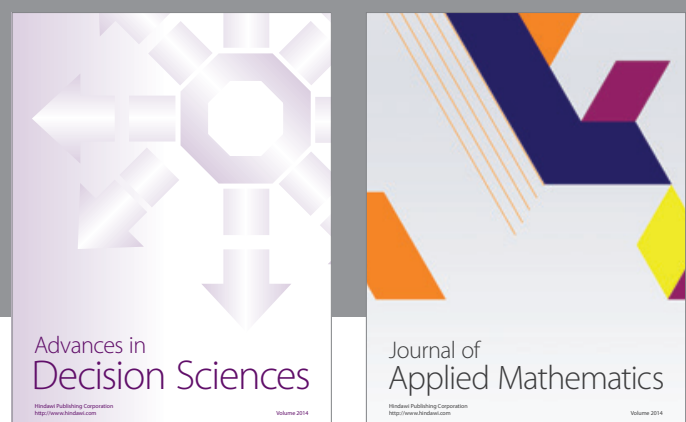

Journal of

Applied Mathematics
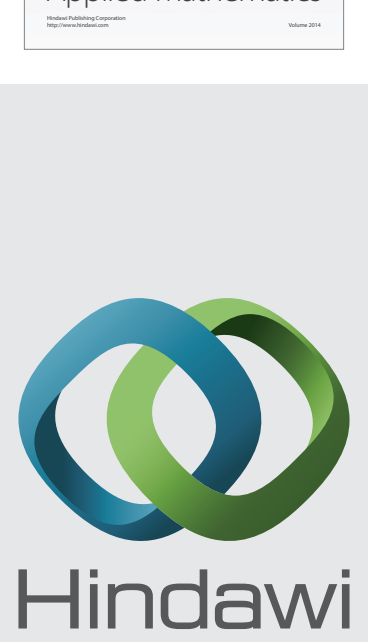

Submit your manuscripts at http://www.hindawi.com
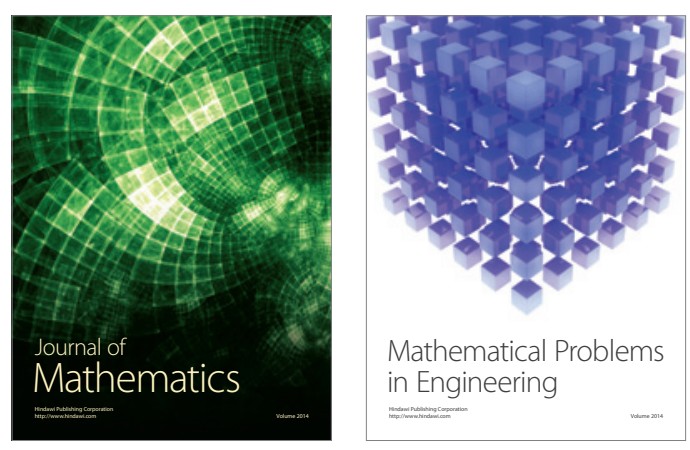

Mathematical Problems in Engineering
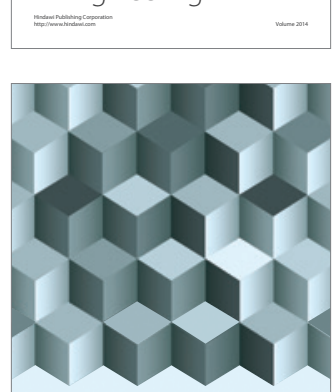

Journal of

Function Spaces
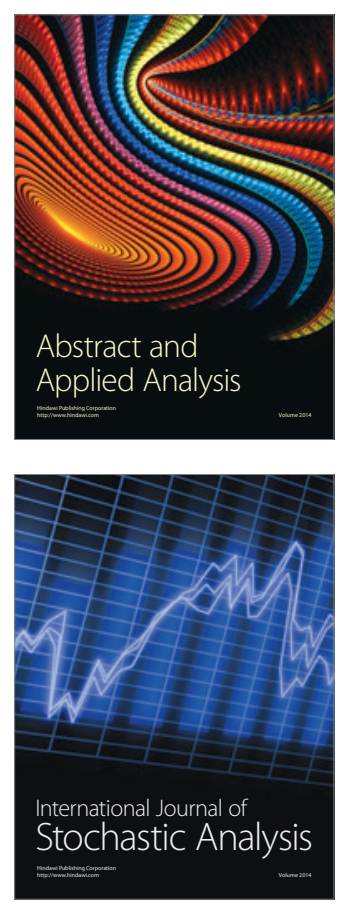

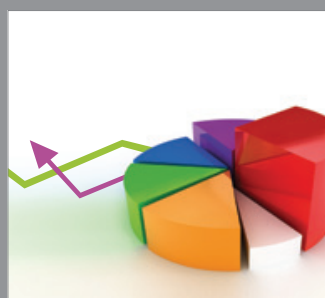

ournal of

Probability and Statistics

Promensencen
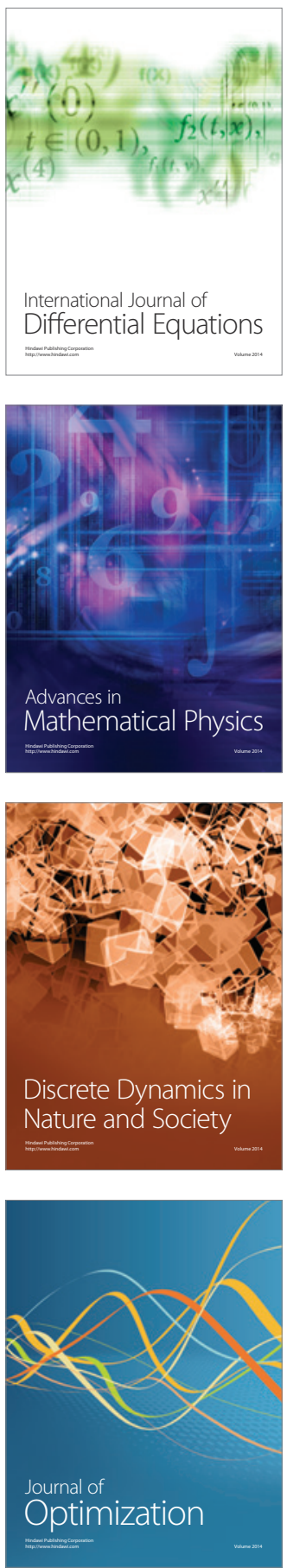